\title{
MAXIMAL FUNCTIONS AND RELATED WEIGHT CLASSES
}

\author{
CARLO SBordone AND IngEmar WiK
}

\begin{abstract}
The famous result of Muckenhoupt on the connection between weights $\omega$ in $A_{p}$-classes and the boundedness of the maximal operator in $L_{p}(\omega)$ is extended to the case $p=\infty$ by the introduction of the geometrical maximal operator. Estimates of the norm of the maximal operators are given in terms of the $A_{p}$-constants. The equality of two differently defined $A_{\infty}$-constants is proved. Thereby an answer is given to a question posed by R. Johnson. For non-increasing functions on the positive real line a parallel theory to the $A_{p}$-theory is established for the connection between weights in $B_{p}$-classes and maximal functions, thereby extending and developing the recent results of Ariño and Muckenhoupt.
\end{abstract}

\section{Introduction}

Let $f$ be a non-negative, locally integrable function defined on $(0, \infty)$. The well-known Carleman inequality (see [4, p. 250])

$$
\int_{0}^{\infty} \exp \left(\frac{1}{x} \int_{0}^{x} \ln f(t) d t\right) d x \leq e \int_{0}^{\infty} f(x) d x,
$$

in which $e$ is the best possible constant, can be considered as the limit case, as $p$ tends to infinity, of the Hardy inequality for $f^{\frac{1}{p}}$

$$
\int_{0}^{\infty}\left(\frac{1}{x} \int_{0}^{x} f(t)^{\frac{1}{p}} d t\right)^{p} d x \leq\left(\frac{p}{p-1}\right)^{p} \int_{0}^{\infty} f(x) d x .
$$

Work supported in part by the National Science Council of Sweden and the Italian Ministero dell'Università e della Ricerca Scientificae Tecnologica. 
In fact, the geometrical mean of $f, \exp \left(\frac{1}{x} \int_{0}^{x} \ln f(t) d t\right.$ ), satisfies (see [4, p. 139])

$$
\lim _{p \rightarrow \infty}\left(\frac{1}{x} \int_{0}^{x} f(t)^{\frac{1}{p}} d t\right)^{p}=\exp \left(\frac{1}{x} \int_{0}^{x} \ln f(t) d t\right) .
$$

We recall that $\left(\frac{p}{p-1}\right)^{p}$ is the best constant in Hardy's inequality and so we deduce

(1.2) $\lim _{p \rightarrow \infty} \sup _{\|g\|_{p}=1} \int_{0}^{\infty}\left(\frac{1}{x} \int_{0}^{x} g(t) d t\right)^{p} d x=\sup _{\|f\|_{1}=1} \int_{0}^{\infty} \exp \left(\frac{1}{x} \int_{0}^{x} \ln f(t) d t\right) d x$.

In the first part of the paper we study analogues of these results in $n$ dimensions for maximal functions and corresponding weights. To be more precise we need some notations.

We let $Q$ stand for a cube with axes parallell to the coordinate axes and $|Q|$ its Lebesgue measure. It is convenient to use a special sign for the mean value over $Q$ of a function $f$

$$
f_{Q} f(x) d x=\frac{1}{|Q|} \int_{Q} f(x) d x .
$$

First we define, for $g \in L_{l o c}^{q}\left(\mathbb{R}^{n}\right), q>0$, the $q$-maximal function of $g$ by

$$
M_{q} g(x)=\sup _{Q \ni x}\left(f|g(t)|_{Q}^{q} d t\right)^{\frac{1}{q}},
$$

where the supremum extends over all cubes $Q \subset \mathbb{R}^{n}$. For $q=1$ we get the familiar Hardy-Littlewood maximal function $M g=M_{1} g$.

As a limit case as $q$ tends to zero, we introduce the geometrical maximal function, $M_{0} g$, by defining

$$
M_{0} g(x)=\sup _{Q \ni x} \exp (f \ln |g(t)| d t) .
$$

For non-increasing, non-negative functions $f$ on $(0, \infty)$ it is easy to show that

$$
M_{\frac{1}{p}} f(x)=\left(\int_{0}^{x} f^{\frac{1}{p}}(t) d t\right)^{p} \text { and } M_{0} f(x)=\exp \int_{0}^{x} \ln f(t) d t
$$


The left and right hand sides of (1.1) therefore are $\lim _{p \rightarrow \infty} M_{\frac{1}{p}} f(x)$ and $M_{0} f(x)$ respectively. We prove in Theorem 2 that

$$
\lim _{p \rightarrow \infty} M_{\frac{1}{p}} f(x)=M_{0} f(x) \text { for } x \in \mathbb{R}^{n} .
$$

The corresponding limit relation to (1.2) will be proved as a corollary to this theorem, but in a much more general situation, where the Lebesgue measure is replaced by a measure $\omega(x) d x$, with $\omega$ a weight in the $A_{\infty^{-}}$class of Muckenhoupt. In section 2.2 we study the limit case as $p$ tends to infinity of the $A_{p}$-constant of a weight function $\omega$.

$$
A_{p}(\omega)=\sup _{Q} f \omega(x) d x\left(f_{Q} \omega^{-\frac{1}{p-1}}(x) d x\right)^{p-1}
$$

and define

$$
A_{\infty}(\omega)=\sup _{Q} f_{Q} \omega(x) d x \exp \left(\int_{Q} \ln \frac{1}{\omega(x)} d x\right), \bar{A}_{\infty}(\omega)=\lim _{p \rightarrow \infty} A_{p}(\omega)
$$

Jensen's inequality implies

$$
A_{\infty}(\omega) \leq \bar{A}_{\infty}(\omega)
$$

Johnson in [6] left it as an open problem whether there exists a constant $c$ such that $\bar{A}_{\infty}(\omega) \leq c A_{\infty}(\omega)$. We settle that problem by showing in Theorem 1, that the two quantities are actually equal.

In Theorem 3 we prove that the geometrical maximal function $M_{0}$ gives a bounded mapping of $L^{1}(\omega)$ into $L^{1}(\omega)$ if and only if the weight function belongs to $A_{\infty}$, thereby extrapolating from $A_{p}$ the classical result of Muckenhoupt [7] on the Hardy-Littlewood maximal function.

In the second part of the paper we restrict our concern to the case of non-increasing, non-negative functions on $(0, \infty)$. Following a recent paper of Ariño and Muckenhoupt [1], we continue to study the classes of weights for which the maximal operator is bounded on non-increasing functions in $L^{p}(\omega)$. It turns out that we have here a more or less complete analogy with the $A_{p}$-classes. Also in this case we study the limit case as $p$ tends to infinity. Our final specialization is to the case when $\omega$ is non-decreasing. 


\section{The general case}

\subsection{Notations and definitions.}

For a non-negative, locally integrable (weight)-function $\omega$ we define $L^{p}(\omega)$ as the class of all measurable functions $f$ such that

$$
\int_{\mathbb{R}^{n}}|f(x)|^{p} \omega(x) d x<\infty, \text { with }\|f\|_{L^{p}(\omega)}=\left(\int_{\mathbb{R}^{n}}|f(x)|^{p} \omega(x) d x\right)^{\frac{1}{p}} .
$$

$A_{p}$ is the class of all weight functions $\omega$ with finite $A_{p}(\omega)$. As $p$ tends to infinity in (1.4) the second factor on the right hand side tends to $\exp \left(f \ln \frac{1}{f(x)} d x\right)$. See [4, p. 71]. It is therefore natural to define the $A_{\infty}$-constant of $\omega$ as

$$
A_{\infty}(\omega)=\sup _{Q} f_{Q} \omega(x) d x \cdot \exp \left(f_{Q} \ln \frac{1}{\omega(x)} d x\right) .
$$

Usually $A_{\infty}$ is not defined as the class of functions for which the right hand side of (2.1) is finite. However, it has been proved by S. V. Hruščev [5], and J. Garcia-Cuerva- R. de Francia [3, p. 405] that this is an alternative definition of $A_{\infty}$.

When studying the boundedness of the maximal operators it is convenient to have the following notations for weight functions $\omega$

$$
m_{p}(\omega)=\sup _{\|f\|_{L(\omega)}=1} \int_{\mathbb{R}^{n}} M_{\frac{1}{p}} f(x) \omega(x) d x .
$$

It is easy to see that

$$
\left.\sup _{\|f\|_{L(\omega)}=1} \int_{\mathbb{R}^{n}} M_{\frac{1}{p}} f(x) \omega(x) d x=\sup _{\|f\|_{L^{p}(\omega)}=1} \int_{\mathbb{R}^{n}}(M f(x))^{p} \omega(x) d x\right) .
$$

We therefore put

$$
m_{\infty}(\omega)=\sup _{\|f\|_{L(\omega)}=1} \int_{\mathbb{R}^{n}} M_{0} f(x) \omega(x) d x .
$$

The non-increasing and non-decreasing rearrangements of a function $f$ will be denoted by $f^{*}$ and $f_{*}$ respectively and are defined by

$$
f^{*}(t)=\sup _{|E|=t} \operatorname{essinf} f(x), \quad f_{*}(t)=\inf _{|E|=t} \operatorname{esssup}_{E} f(x)
$$

and then become continuous to the right and left respectively. 


\section{2. $A_{\infty}$ as a limit case of $A_{p}$.}

It is well known that $A_{\infty}$ can be defined as $\bigcup_{p>1} A_{p}$. Let $\omega$ be a function in $A_{\infty}$. Then there exists a number $p_{1}, 1 \leq p_{1}<\infty$ such that $\omega \in A_{p}$ for $p \geq p_{1}$. Since, by Hölder's inequality, $A_{p}(\omega)$ is a decreasing function of $p$, we have two candidates for the $A_{\infty}$-constant of $\omega$, namely

$$
\bar{A}_{\infty}(\omega)=\lim _{p \rightarrow \infty} A_{p}(\omega) \quad \text { and } \quad A_{\infty}(\omega) \quad \text { as defined by (2.1). }
$$

By Jensen's inequality

$$
\exp \left(f_{Q} \ln g(x) d x\right) \leq \int_{Q} g(x) d x .
$$

We apply this with $g=\omega^{-\frac{1}{p}}$, raise both sides to the power $p$ and obtain

$$
\exp \left(f_{Q} \ln \frac{1}{\omega(x)} d x\right) \leq\left(f_{Q} \frac{d x}{\omega(x)^{\frac{1}{p}}}\right)^{p}
$$

which means that $A_{\infty}(\omega) \leq A_{p+1}(\omega)$ and thus $A_{\infty}(\omega) \leq \bar{A}_{\infty}(\omega)$. It has been an open question, [6, p. 98], whether there exists a constant $c$ such that $\bar{A}_{\infty}(\omega) \leq c A_{\infty}(\omega)$. Here is the answer.

Theorem 1. If $\omega \in A_{\infty}$, then $A_{\infty}(\omega)=\bar{A}_{\infty}(\omega)$.

In the proof of the theorem we will use the following lemma.

Lemma 1. Let $f$ be a non-negative integrable function on $(0,1)$ and $p$ a real number, $p \geq 1$. Put

$$
g(x)= \begin{cases}f(x), & \text { if } f(x)<e \\ e, & \text { elsewhere }\end{cases}
$$

Then

$$
\left(\int_{0}^{1} f(x) d x\right)^{p}-\left(\int_{0}^{1} g(x) d x\right)^{p} \leq p\left(\int_{E} f(x) d x\right) \cdot\left(\int_{0}^{1} f(x) d x\right)^{p-1}
$$

where $E=\{x \in(0,1) ; f(x) \geq e\}$. 
Proof: This is an immediate consequence of the inequality

$$
b^{p}-a^{p} \leq p(b-a) b^{p-1}, \quad \text { for } 0 \leq a \leq b, \quad p \geq 1,
$$

with

$$
b=\int_{0}^{1} f(x) d x \text { and } \quad a=\int_{0}^{1} g(x) d x
$$

Proof of Theorem 1: Suppose $\omega$ is a function in $A_{p_{1}}\left(\mathbb{R}^{n}\right)$ with $A_{p_{1}}$ constant $A$. We will show that, for every $p \geq p_{1}-1$, we have

$$
\sup _{Q} f_{Q} \omega(x) d x \cdot\left(\int_{Q} \frac{d x}{\omega(x)^{\frac{1}{p}}}\right)^{p} \leq\left(1+\delta\left(p, p_{1}, A\right)\right) \cdot A_{\infty}(\omega)
$$

where $\lim _{p \rightarrow \infty} \delta\left(p, p_{1}, A\right)=0$. This implies $\bar{A}_{\infty}(\omega) \leq A_{\infty}(\omega)$ which proves the theorem.

Except for the supremum the left hand side of (2.5) is invariant under changes of scale in $\mathbb{R}^{n}$ and also under multiplication of $\omega$ by a positive constant. Without loss of generality we may therefore assume that that $|Q|=1$ and $\omega(Q)=\int_{Q} \omega(x) d x=1$.

We denote here by $\omega_{*}(t), \quad 0 \leq t \leq|Q|=1$ the non-decreasing rearrangement of the restriction of $\omega$ to $Q$. Then, from [10, p. 250] e.g., and the definition of $A_{\infty}(\omega)$, we conclude that

$$
\omega_{*}(t) \geq A^{-1} t^{p_{1}-1} \quad \text { and } \quad \exp \left(\int_{0}^{1} \ln \frac{1}{\omega_{*}(t)} d t\right) \leq A_{\infty}(\omega) .
$$

Since

$$
e^{x} \leq 1+x+x^{2}, \text { for } \quad x \leq 1
$$

we have

$$
\int_{0}^{1} \frac{d t}{\omega_{*}(t)^{\frac{1}{p}}}=\int_{0}^{1} \exp \left(\frac{1}{p} \ln \frac{1}{\omega_{*}(t)}\right) d t \leq \int_{0}^{1}\left(1+\frac{1}{p} \ln \frac{1}{\omega_{*}(t)}+\frac{1}{p^{2}}\left(\ln \frac{1}{\omega_{*}(t)}\right)^{2}\right) d t,
$$

if $\omega_{*}(t) \geq e^{-p}$. This means that

$$
\left(\int_{0}^{1} \frac{d t}{\omega_{*}(t)^{\frac{1}{p}}}\right)^{p} \leq \exp p \ln \left(1+\frac{1}{p} \int_{0}^{1} \ln \frac{1}{\omega_{*}(t)} d t+\frac{1}{p^{2}} \int_{0}^{1}\left(\ln \frac{1}{\omega_{*}(t)}\right)^{2} d t\right) .
$$


The inequality: $\ln (1+x) \leq x$, for $x>-1$, implies

$$
\left(\int_{0}^{1} \frac{d t}{\omega_{*}(t)^{\frac{1}{p}}}\right)^{p} \leq \exp \left(\int_{0}^{1} \ln \frac{1}{\omega_{*}(t)} d t\right) \cdot \exp \left(\frac{1}{p} \int_{0}^{1}\left(\ln \frac{1}{\omega_{*}(t)}\right)^{2} d t\right) .
$$

We now use (2.6) to find

$$
\int_{0}^{1}\left(\ln \frac{1}{\omega_{*}(t)}\right)^{2} d t \leq \int_{0}^{1}\left(\ln A+\left(p_{1}-1\right) \ln \frac{1}{t}\right)^{2} d t=c\left(p_{1}, A\right) .
$$

The second factor on the right hand side of (2.7) therefore converges to 1 as $\mathrm{p}$ tends to infinity. This proves the theorem for functions $\omega$ that are bounded below by a positive constant a. (We just choose p so large that $e^{-p}<a$.) If that is not the case we construct a new function

$$
\omega_{p}(x)= \begin{cases}\omega(x), & \text { if } \omega(x)>e^{-p} \\ e^{-p}, & \text { if } \omega(x) \leq e^{-p}\end{cases}
$$

It is easy to check that (2.7) is valid with $\omega_{*}$ replaced by $\left(\omega_{p}\right)_{*}$. After the replacement we increase the right hand side of (2.7) and use the second inequality of (2.6) to find

$$
\begin{aligned}
&\left(\int_{0}^{1} \frac{d t}{\left(\omega_{p}\right)_{*}(t)^{\frac{1}{p}}}\right)^{p} \leq \exp \left(\int_{0}^{1} \ln \frac{1}{\omega_{*}(t)} d t\right) \cdot \exp \left(\frac{1}{p} \int_{0}^{1}\left(\ln \frac{1}{\omega_{*}(t)}\right)^{2} d t\right) \leq \\
& \leq A_{\infty}(\omega) \cdot \exp \left(\frac{c\left(p_{1}, A\right)}{p}\right)
\end{aligned}
$$

We now take a closer look at the left hand side of this inequality. We want to replace $\left(\omega_{p}\right)_{*}$ by $\omega_{*}$ and estimate the difference in a way that is independent of our particular choice of cube $Q$. For this we will use Lemma 1, applied with $f(x)=\omega_{*}(t)^{-\frac{1}{p}}$. From (2.6) we conclude that

$$
\omega_{*}(t)^{-\frac{1}{p}}>e \Longrightarrow A^{-1} t^{p_{1}-1}<e^{-p} \Longrightarrow t<A^{\frac{1}{p_{1}-1}} e^{-\frac{p}{p_{1}-1}}=t_{p} .
$$

Hence $E \subset\left(0, A^{\frac{1}{p_{1}-1}} e^{-\frac{p}{p_{1}-1}}\right)$ in the lemma and we have the estimate

$$
\begin{array}{r}
\int_{E} \frac{d t}{\omega_{*}(t)^{\frac{1}{p}}} \leq A^{\frac{1}{p}} \int_{0}^{t_{p}} \frac{d t}{t^{\frac{p_{1}-1}{p}}}=\frac{A^{\frac{1}{p}} t_{p}^{1-\frac{p_{1}-1}{p}}}{\left(1-\frac{p_{1}-1}{p}\right)}=\frac{p}{p-p_{1}+1} A^{\frac{1}{p_{1}-1}} e^{-\frac{p}{p_{1}-1}+1}= \\
=c\left(p, p_{1}, A\right) .
\end{array}
$$


This estimate is used in (2.8) and, combined with Lemma 1, the conclusion is

$$
\left(\int_{0}^{1} \frac{d t}{\omega_{*}(t)^{\frac{1}{p}}}\right)^{p} \leq A_{\infty}(\omega) \cdot \exp \frac{c\left(p_{1}, A\right)}{p}+d\left(p, p_{1}, A\right)\left(\int_{0}^{1} \frac{d t}{\omega_{*}(t)^{\frac{1}{p}}}\right)^{p-1}
$$

where $d\left(p, p_{1}, A\right)=p \cdot c\left(p, p_{1}, A\right)$. This quantity obviously tends to zero as $p$ tends to infinity.

We now take an arbitrary $\epsilon, 0<\epsilon<1$, and choose $p$ so large that $\exp \frac{c\left(p_{1}, A\right)}{p} \leq(1+\epsilon)$ and $d\left(p, p_{1}, A\right) \leq \epsilon$. Put $v=\left(\int_{0}^{1} \frac{d t}{\omega_{*}(t)^{\frac{1}{p}}}\right)^{p}$. Then $v \geq 1$ by Hölder's inequality and

$$
v \leq(1+\varepsilon) A_{\infty}(\omega)+\varepsilon v^{\frac{p-1}{p}} \leq(1+\varepsilon) A_{\infty}(\omega)+\varepsilon v,
$$

i.e.

$$
v \leq \frac{1+\varepsilon}{1-\varepsilon} A_{\infty}(\omega)<(1+3 \varepsilon) A_{\infty}(\omega)
$$

Now we take supremum over all cubes $Q$ and get

$$
A_{p+1}(\omega)=\left(1+\delta\left(p, p_{1}, A\right)\right) \cdot A_{\infty}(\omega) \leq(1+3 \varepsilon) A_{\infty}(\omega)
$$

where $\delta\left(p, p_{1}, A\right)$ tends to zero as $p$ tends to infinity. This means that

$$
\lim _{p \rightarrow \infty} A_{p}(\omega) \leq A_{\infty}(\omega)
$$

which concludes the proof of the theorem.

It is also possible to have an estimate of the rate of convergence. A simple analysis of the various inequalities will give us the estimate

$$
\delta\left(p, p_{1}, A\right) \leq \frac{C\left(p_{1}, A\right)}{p}
$$

where $C\left(p_{1}, A\right)$ is a constant depending only on $p_{1}$ and $A$. 


\section{3. $M_{0}$ as a limit case of $M_{\frac{1}{p}}$.}

Corresponding to the preceding paragraph, we present here a result on the geometrical maximal function, $M_{0} f$, the precise importance of which is demonstrated in Theorem 3 at the end of this paragraph.

Theorem 2. Suppose that $f$ lies in $L_{\text {loc }}^{\alpha}\left(\mathbb{R}^{n}\right)$, for some $\alpha>0$. Then we have

$$
\lim _{p \rightarrow \infty} M_{\frac{1}{p}} f(x)=M_{0} f(x), \quad \forall x .
$$

Proof: By Jensen's inequality

$$
\exp f_{Q} \ln f(x) d x \leq\left(\int_{Q} f^{\frac{1}{p}}(x) d x\right)^{p} .
$$

We take the supremum over all $Q$ that contain $x$ and obtain

$$
M_{0} f(x) \leq M_{\frac{1}{p}} f(x), \quad \forall x,
$$

and letting $p$ tend to infinity this gives

$$
M_{0} f(x) \leq \lim _{p \rightarrow \infty} M_{\frac{1}{p}} f(x), \quad \forall x .
$$

It remains to prove the opposite inequality of (2.9). We assume first that the number $\alpha$ in the theorem equals one. Then we use Lemma 2 below, according to which we have, for every $\epsilon \in(0,1)$ and cube $Q$ :

$$
\left(\int_{Q} f^{\frac{1}{p}}(x) d x\right)^{p} \leq \exp f_{Q} \ln f_{\epsilon}(x) d x \cdot \exp \frac{(\ln \epsilon)^{2}+1}{p}+\frac{p}{e^{p}-1} \int_{Q} f(x) d x,
$$

where

$$
f_{\epsilon}(x)= \begin{cases}f(x), & \text { if } f \geq \epsilon f f(x) d x \\ f f(x) d x & \text { elsewhere. }\end{cases}
$$

From this we conclude

$$
\sup _{Q \ni x}\left(f_{Q} f^{\frac{1}{p}}(t) d t\right)^{p} \leq \sup _{Q \ni x}\left(\exp \int_{Q} \ln f_{\epsilon}(t) d t \cdot \exp \frac{(\ln \epsilon)^{2}+1}{p}+\frac{p}{e^{p}-1} f_{Q} f(t) d t\right) .
$$

$f_{\epsilon}$ is independent of $p$. Letting $p$ tend to infinity therefore gives us

$$
\lim _{p \rightarrow \infty} M_{\frac{1}{p}} f(x) \leq \sup _{Q \ni x} \exp \left(f_{Q} \ln f_{\epsilon}(t) d t\right) .
$$


Now we let $\epsilon$ tend to zero. By monotone convergence

$$
\lim _{p \rightarrow \infty} M_{\frac{1}{p}} f(x) \leq M_{0} f(x), \quad \forall x .
$$

This concludes the proof if $\alpha=1$. For $\alpha \neq 1$ we put $g=f^{\alpha}$ and use (2.11) on $g$. This gives

$$
\lim _{p \rightarrow \infty} \sup _{Q \ni x}\left(f_{Q} f^{\frac{\alpha}{p}}(t) d t\right)^{p} \leq \sup _{Q \ni x} \exp f_{Q} \ln f^{\alpha}(t) d t
$$

or, with $q=p \alpha^{-1}$,

$$
\lim _{q \rightarrow \infty} M_{\frac{1}{q}} f(x) \leq M_{0} f(x), \quad \forall x .
$$

This is (2.11), which thus is valid for all $\alpha>0$. Combined with (2.9) this gives the desired equality.

What remains of the proof therefore is the main step, namely to prove the lemma.

Lemma 2. Suppose that $f$ is a locally integrable function, defined on $\mathbb{R}^{n}$. Then (2.10) is valid for every $\epsilon \in(0,1)$ and every cube $Q$ in $\mathbb{R}^{n}$.

Proof: The homogenity of (2.10) allows us to assume that $|Q|=1$ and $f_{Q} f(x) d x=1$. We may, by turning to the non-increasing rearrangement of the restriction of $f$ to $Q$, even assume that we are dealing with a nonincreasing function on $(0,1)$. This means that it is sufficient to prove that if $\epsilon \in(0,1)$ and $\int_{0}^{1} f(x) d x=1$ then

$$
\left(\int_{0}^{1} f^{\frac{1}{p}}(x) d x\right)^{p} \leq \exp \int_{0}^{1} \ln f_{\epsilon}(x) d x \cdot \exp \frac{(\ln \epsilon)^{2}+1}{p}+\frac{p}{e^{p}-1}
$$

where

$$
f_{\epsilon}(x)= \begin{cases}f(x), & \text { if } f \geq \epsilon \\ 1 & \text { elsewhere }\end{cases}
$$

Put

$$
E_{\epsilon}=\{x \in(0,1) ; f(x) \geq \epsilon\} \text { and }\left|E_{\epsilon}\right|=1-l(\epsilon) .
$$

We first assume that $0 \leq f(x) \leq e^{p}$ on $(0,1)$. Since $f^{\frac{1}{p}}(x)=\exp \frac{\ln f(x)}{p}$ we can use the inequality $e^{x} \leq 1+x+x^{2}$, for $x \leq 1$, to find

$$
\begin{aligned}
\left(\int_{0}^{1} f_{\epsilon}^{\frac{1}{p}}(x) d x\right)^{p} \leq\left(\epsilon^{\frac{1}{p}} l(\epsilon)+\int_{E_{\epsilon}} f^{\frac{1}{p}}(x) d x\right)^{p} & \leq\left(\left(\epsilon^{\frac{1}{p}}-1\right) l(\epsilon)+1+\right. \\
& \left.+\int_{E_{\epsilon}} \frac{\ln f}{p} d x+\int_{E_{\epsilon}} \frac{(\ln f)^{2}}{p^{2}} d x\right)^{p} .
\end{aligned}
$$


By assumption $\epsilon^{\frac{1}{p}}-1<0$ and by definition $f_{\epsilon} \geq f$. Thus

$$
\begin{aligned}
\left(\int_{0}^{1} f^{\frac{1}{p}}(x) d x\right)^{p} \leq\left(1+\int_{E_{\epsilon}} \frac{\ln f}{p} d x\right. & \left.+\int_{E_{\epsilon}} \frac{(\ln f)^{2}}{p^{2}} d x\right)^{p}= \\
& =\left(1+\int_{0}^{1} \frac{\ln f_{\epsilon}}{p} d x+\int_{0}^{1} \frac{\left(\ln f_{\epsilon}\right)^{2}}{p^{2}} d x\right)^{p} .
\end{aligned}
$$

What is inside the last parenthesis obviously is positive and we can use the inequality: $\ln (1+x) \leq x$ for $x>-1$, to obtain

$$
\left(\int_{0}^{1} f^{\frac{1}{p}}(x) d x\right)^{p} \leq \exp \int_{0}^{1} \ln f_{\epsilon}(x) d x \cdot \exp \frac{1}{p} \int_{0}^{1}\left(\ln f_{\epsilon}(x)\right)^{2} d x .
$$

It is easy to see that $(\ln t)^{2}<t$ if $t>1$. Therefore

$$
\int_{0}^{1}\left(\ln f_{\epsilon}\right)^{2} d x \leq(\ln \epsilon)^{2}\left|E_{\epsilon}\right|+\int_{0}^{1} f(x) d x \leq(\ln \epsilon)^{2}+1 .
$$

When we plug that into formula (2.12) we get something which is a little stronger than $\left(2.10^{\prime}\right)$. However, we have to get rid of our extra assumption that $f<e^{p}$ on $(0,1)$. We consider the truncated function

$$
g_{p}(x)= \begin{cases}f(x), & \text { if } f(x)<e^{p} \\ e^{p}, & \text { elsewhere }\end{cases}
$$

We can apply exactly the same arguments as before to the function $g_{p}$ and obtain

$$
\left(\int_{0}^{1} g_{p}^{\frac{1}{p}}(x) d x\right)^{p} \leq\left(\exp \left(\int_{E_{e}} \ln g_{p} d x\right)\right) \cdot \exp \frac{(\ln \epsilon)^{2}+1}{p} .
$$

Since $g_{p}(x) \leq f(x)$, we can replace $g_{p}$ by $f$ on the right hand side. To estimate the left hand side we use Lemma 1 with $f(x)$ replaced by $f^{\frac{1}{p}}(x)$. Then $g(x)$ of the lemma will be $g_{p}^{\frac{1}{p}}(x)$ and the result

$$
\left(\int_{0}^{1} f^{\frac{1}{p}}(x) d x\right)^{p} \leq\left(\int_{0}^{1} g_{p}^{\frac{1}{p}}(x) d x\right)^{p}+p \int_{0}^{x_{p}} f^{\frac{1}{p}}(x) d x \cdot\left(\int_{0}^{1} f^{\frac{1}{p}}(x) d x\right)^{p-1}
$$


where $x_{p}=\sup \left\{x \in(0,1) ; f(x)>e^{p}\right\}$. For $x \in\left(0, x_{p}\right)$ we have

$$
f^{\frac{1}{p}}(x)=\frac{f(x)}{f(x)^{1-\frac{1}{p}}} \leq \frac{f(x)}{e^{p-1}}
$$

which, when integrated, gives

$$
\int_{0}^{x_{p}} f^{\frac{1}{p}}(x) d x \leq \frac{1}{e^{p-1}}
$$

Also, by Hölder's inequality,

$$
\int_{0}^{1} f^{\frac{1}{p}}(x) d x \leq\left(\int_{0}^{1} f(x) d x\right)^{\frac{1}{p}}=1
$$

Therefore

$$
\left(\int_{0}^{1} f^{\frac{1}{p}}(x) d x\right)^{p} \leq\left(\int_{0}^{1} g_{p}^{\frac{1}{p}}(x) d x\right)^{p}+\frac{p}{e^{p-1}},
$$

which, combined with (2.13) gives

$$
\left(\int_{0}^{1} f^{\frac{1}{p}}(x) d x\right)^{p} \leq\left(\exp \left(\int_{0}^{1} \ln f_{\epsilon}(x) d x\right)\right) \cdot \exp \frac{(\ln \epsilon)^{2}+1}{p}+\frac{p}{e^{p-1}} .
$$

So we have proved $\left(2.10^{\prime}\right)$ and the proof is complete.

\section{Corollary.}

$$
\lim _{p \rightarrow \infty} m_{p}(\omega)=m_{\infty}(\omega)
$$

Proof: Choose an arbitrary $\epsilon>0$. As an immediate consequence of Hölder's inequality and the monotone convergence theorem there exists, for every $f$, a number $p_{0}$, such that

$$
\left|\int_{\mathbb{R}^{n}} M_{\frac{1}{p_{0}}} f(x) \omega(x) d x-\int_{\mathbb{R}^{n}} M_{0} f(x) \omega(x) d x\right|<\epsilon .
$$

In particular we can take an $f$ with $\|f\|_{L(\omega)}=1$ such that the second integral differs from $m_{\infty}(\omega)$ with at most $\epsilon$. Since $M_{\frac{1}{p}} f \geq M_{0} f$ we obviously have

$$
m_{\infty}(\omega) \leq m_{p_{0}}(\omega) \leq m_{\infty}(\omega)+2 \epsilon
$$


However, $\epsilon>0$ is arbitrary and we obtain (2.14).

Muckenhoupt [7, p. 222] has shown that the maximal operator $M$ gives a bounded mapping from $L^{p}(\omega)$ to $L^{p}(\omega)$ if and only if $\omega \in A_{p}$. In other words :

$$
\left.\omega \in A_{p} \Longleftrightarrow \sup _{\|f\|_{L^{p}(\omega)}=1} \int_{\mathbb{R}^{n}}(M f(x))^{p} \omega(x) d x\right)<\infty .
$$

Put here $g=f^{p}$ and take into account that $M_{\frac{1}{p}} g=\left(M g^{\frac{1}{p}}\right)^{p}$. Then, using our terminology (1.4) and (2.2), Muckenhoupt's result can be rephrased as

Theorem M. A weight function $\omega$ is in $A_{p}$ if and only if

$$
m_{p}(\omega)=\sup _{\|f\|_{L(\omega)}=1} \int_{\mathbb{R}^{n}} M_{\frac{1}{p}} f(x) \omega(x) d x<\infty
$$

and we have

$$
A_{p}(\omega) \leq m_{p}(\omega) \leq g\left(A_{p}(\omega), p, n\right)
$$

In the theorem below we will show that the limit case, $p=\infty,\left(M_{\frac{1}{p}}\right.$ replaced by $M_{0}$ ), of this theorem is true. Furthermore, we will give an estimate of $m_{\infty}(\omega)$ in terms of the $A_{\infty}$-constant of $\omega$.

Theorem 3. A weight function $\omega$ is in $A_{\infty}$ if and only if

$$
m_{\infty}(\omega)=\sup _{\|f\|_{L(\omega)}=1} \int_{\mathbb{R}^{n}} M_{0} f(x) \omega(x) d x<\infty .
$$

and we have

$$
A_{\infty}(\omega) \leq m_{\infty}(\omega) \leq C_{1}(n)\left(A_{\infty}(\omega)\right)^{150 n}
$$

where $C_{1}(n)$ is a constant, depending only on $n$. 
Proof: For the sufficiency we just note that, by the corollary above, $m_{\infty}(\omega)<\infty$ implies $m_{p}(\omega)<\infty$ for $p$ large enough and by Theorem M it follows that $\omega \in A_{p}$ for $p$ large enough and

$$
A_{p}(\omega) \leq m_{p}(\omega)
$$

The sufficiency part and the first inequality of the theorem now follow from (2.14) and Theorem 1 by letting $p$ tend to infinity in this formula.

For the necessity part we assume that $\omega$ is in $A_{\infty}$ with $A_{\infty}(\omega)=A$. We use the result by Hrušcev [5, p. 255], according to which, for a subset $E$ of any cube $Q$, we have

$$
\frac{|E|}{|Q|} \geq \frac{1}{2} \Longrightarrow \frac{\omega(E)}{\omega(Q)} \geq \frac{1}{1+4 A^{2}} \Longrightarrow \frac{\omega(E)}{\omega(Q)} \geq \frac{1}{5 A^{2}} .
$$

Now we can use the estimate in theorem 3 of $[10$, p. 252] to deduce that for $\beta>(n+2) \log _{2}\left(5 A^{2}\right)=\beta_{0}$ we have, for any $E \subset Q$

$$
\frac{\omega(E)}{\omega(Q)} \geq \frac{1}{5 A^{2}}\left(\frac{|E|}{|Q|}\right)^{\beta} .
$$

According to corollary 1, p. 250 of the same paper this implies that $\omega$ is in $A_{p}$ for $p>\beta_{0}$ and with

$$
A_{p}(\omega) \leq\left(5 A^{2}\right)\left(\frac{p-1}{p-\beta_{0}}\right)^{p-1} \leq 5 A^{2} e^{2 \beta_{0}} \leq\left(5 A^{2}\right)^{3 n+7}=\dot{B}
$$

for $p>3 \beta_{0}$.

Buckley, $[\mathbf{2}$, p. 9], has shown that the maximal operator is of weak type $(p, p)$ on $L^{p}(\omega)$ with weak-norm $\left(C(n) A_{p}(\omega)\right)^{\frac{1}{p}}$. We use this result and Marcinkiewicz interpolation theorem (see Torchinsky [9, p. 87]) to interpolate in the interval $\left(p_{0}=\right) 3 \beta_{0}<2 p_{0}<\infty$ and find that

$$
m_{2 p_{0}} \leq\left(8 e^{\frac{1}{e}}\right)^{2 p_{0}} C(n)^{2} B p_{0}^{-1}
$$

Taking into account $p_{0}=3 \beta_{0}$ and the definition (2.17) of $B$, this implies

$$
m_{p}(\omega) \leq C_{1}(n) A^{50 n+100} \text { for } \quad p \geq 3 \beta_{0} .
$$

Hence

$$
m_{\infty}(\omega) \leq C_{1}(n)\left(A_{\infty}(\omega)\right)^{150 n}
$$




\section{The case of non-increasing functions on $(0, \infty)$.}

\subsection{Notations and definitions.}

For non-negative, non-increasing functions on $(0, \infty)$ the maximal functions $M_{q} f$ and $M_{0} f$ satisfy

$$
M_{q} f(x)=\left(\int_{0}^{x} f^{q}(t) d t\right)^{\frac{1}{q}} \quad \text { and } \quad M_{0} f(x)=\exp \int_{0}^{x} \ln f(t) d t .
$$

Ariño and Muckenhoupt [1, p. 727-734] have shown that in this case and for $1 \leq p<\infty$ a necessary and sufficient condition on $\omega$ to secure that there exists a constant $C$, such that

$$
\int_{0}^{\infty}(M f(x))^{p} \omega(x) d x \leq C \int_{0}^{\infty} f^{p}(x) \omega(x) d x
$$

is valid, for all non-negative, non-increasing functions in $L^{p}(\omega)$ on $(0, \infty)$, is the existence of a constant $B$, such that

$$
\int_{x}^{\infty} \frac{\omega(t)}{t^{p}} d t \leq \frac{B}{x^{p}} \int_{0}^{x} \omega(t) d t, \quad \forall x>0 .
$$

They also proved that a sufficient condition on $\omega$ is

$$
\sup _{x>0}\left[f \int_{0}^{x} \omega(t) d t\right]\left[\int_{0}^{x}(\omega(t))^{-\frac{1}{p-1}} d t\right]^{p-1}=A_{p}^{\prime}(\omega)<\infty,
$$

and that this condition is also necessary if the weight function $\omega$ is non decreasing.

We will denote by $B_{p}, 0<p<\infty$ and $A_{p}^{\prime}, 1 \leq p<\infty$ the class of all functions $\omega$ satisfying (3.2) and (3.3) respectively. (For $p=1$ the second factor to the left in (3.3) should be interpreted as $\underset{0<t<x}{\operatorname{ess} \sup } \frac{1}{\omega(t)}$. $)$ We also say that $\omega$ lies in $B_{p}$ with constant $B_{p}(\omega)$ if $B_{p}(\omega)$ is the minimal constant for which (3.2) is valid. Let $p$ tend to infinity in (3.3). This natural way leads us to the definition of $A_{\infty}^{\prime}$ as those non-negative, measurable functions $\omega$ that satisfy

$$
\sup _{x>0}\left[\int_{0}^{x} \omega(t) d t\right]\left[\exp \int_{0}^{x} \ln \frac{1}{(\omega(t)} d t\right]=A_{\infty}^{\prime}(\omega)<\infty .
$$


In analogy with the $n$-dimensional case we define, for $p>0$

$$
m_{p}^{\prime}(\omega)=\sup \int_{0}^{\infty} M_{\frac{1}{p}} f(x) \omega(x) d x
$$

but now the supremum is taken over all non-increasing $f$ on $(0, \infty)$ with $\|f\|_{L(\omega)}=1$. We note that $m_{p}^{\prime}(\omega)$ is the infimum of all $C$ such that (3.1) holds. Correspondingly we define

$$
m_{\infty}^{\prime}(\omega)=\sup _{f} \int_{0}^{\infty} M_{0} f(x) \omega(x) d x
$$

where the supremum is taken over the same class.

3.2. The analogy between $A_{p}$ and $B_{p}$.

In Lemma (2.1) of [1] there is a proof, of the fact that $\omega \in B_{p}$ implies that $\omega \in B_{p-\epsilon}$ for some $\epsilon>0$ (a similar result is in Strömberg-Torchinsky $[8$, p. 12]). We give here a short and sharp proof of that lemma.

Lemma 3. Suppose that $0<p<\infty$ and $\omega$ is a function in $B_{p}$ such that

$$
\int_{x}^{\infty} \frac{\omega(t)}{t^{p}} d t \leq \frac{B}{x^{p}} \int_{0}^{x} \omega(t) d t, \quad \forall x>0 .
$$

Then $\omega \in B_{p-\epsilon}$ for $\epsilon<\frac{p}{B+1}$ i.e. $\omega \in B_{p_{1}}$ for $p_{1}<\frac{B}{B+1} p$ and $B_{p}(\omega) \leq$ $\frac{B p}{p-\epsilon(B+1)}$. The upper bound of $\epsilon$ is best possible.

Proof: Choose $\epsilon<\frac{p}{B+1}$, multiply (3.4) by $x^{\epsilon-1}$ and integrate from $r$ to infinity. A change of the order of integration on both sides then results in

$$
\frac{1}{\epsilon} \int_{r}^{\infty} \frac{\omega(t)}{t^{p}}\left(t^{\epsilon}-r^{\epsilon}\right) d t \leq \frac{B}{p-\epsilon}\left(\int_{0}^{r} \frac{\omega(t)}{r^{p-\epsilon}} d t+\int_{r}^{\infty} \frac{\omega(t)}{t^{p-\epsilon}} d t\right),
$$

which gives us, after once more using (3.4)

$$
\begin{aligned}
&\left(\frac{1}{\epsilon}-\frac{B}{p-\epsilon}\right) \int_{r}^{\infty} \frac{\omega(t)}{t^{p-\epsilon}} d t \leq \frac{r^{\epsilon}}{\epsilon} \int_{r}^{\infty} \frac{\omega(t)}{t^{p}} d t+\frac{B}{(p-\epsilon) r^{p-\epsilon}} \int_{0}^{r} \omega(t) d t \leq \\
& \leq\left(\frac{1}{\epsilon}+\frac{1}{p-\epsilon}\right) \frac{B}{r^{p-\epsilon}} \int_{0}^{r} \omega(t) d t .
\end{aligned}
$$


This is to say that $\omega \in B_{p-\epsilon}$ for $\epsilon<\frac{p}{B+1}$ and $B_{p}(\omega) \leq \frac{B p}{p-\epsilon(B+1)}$.

To show that the limit is best possible we just take $\omega(x)=x^{\alpha}, \alpha>-1$, and $p>\alpha+1$. Then the $B_{p}$-constant of $\omega$ is $\frac{\alpha+1}{p-\alpha-1}$. By the result above we see that $\omega \in B_{p_{1}}$ for

$$
p_{1}>p-\frac{p}{\frac{\alpha+1}{p-\alpha-1}+1}=\alpha+1
$$

Of course no smaller $p$ 's are possible, if the left side of (3.6) is to converge.

We will extend the results of [1] to the geometrical maximal function $M_{0} f$ (and also in some cases to $0<p \leq 1$. To make apparent the parallellity with the ordinary $A_{p}$-classes, we introduce a class $B_{\infty}$. It will soon become evident that the corresponding to the definition of $A_{\infty}$ would be to define $B_{\infty}$ as the class of weight functions, for which there exist two constants $r<1$ and $k>0$ such that

$$
1>\frac{t}{x} \geq r \Longrightarrow \frac{\int_{0}^{t} \omega(u) d u}{\int_{0}^{x} \omega(u) d u} \geq k .
$$

This is equivalent to the following definition, which is more easy to grasp.

Definition. $B_{\infty}$ is the class of non-negative, locally integrable functions $\omega$ on $(0, \infty)$ with the property that there exist two constants $r, 0<r<1$ and $C>0$ such that

$$
C \int_{0}^{r x} \omega(t) d t \geq \int_{0}^{x} \omega(t) d t, \quad \forall x>0 .
$$

Remark. We could equally well have made the definition with $r=\frac{1}{2}$ instead of being arbitrary. This would seemingly be more restrictive for $r>\frac{1}{2}$. However if $\omega$ satisfies our definition with an $r>\frac{1}{2}$ we can iterate the inequality approximately $\left(-\log _{2} r\right)^{-1}$ of times to see that it is satisfied for $r=\frac{1}{2}$, but with a larger $C$.

Definition. The doubling constant, $d(\omega)$ is the minimum of all $C$ such that (3.5) is valid with $r=\frac{1}{2}$. If $d(\omega)$ is finite we will say that $\omega$ has the doubling property.

It is immediately evident from the definition that $B_{p_{1}} \subset B_{p}$ and also that $B_{p}(\omega) \leq B_{p_{1}}(\omega)$ if $p_{1}<p$. 
A function in $B_{p}$ obviously has the doubling property. (Just relax in the definition (3.2) by reducing the interval of integration on the left in (3.2) to become $(x, 2 x)$. However, we can do much better and obtain an estimate of $C$ in (3.5), an estimate that depends on $r$ and also can be used as an alternative characterization of $B_{p}$. (Compare corollary $1, \mathrm{p}$. 250 of $[10]$.)

Theorem 4. A weight function $\omega$ is in $B_{p}$, if and only if there exist constants $p_{1}, 0<p_{1}<p$, and $C$ such that

$$
\int_{0}^{t} \omega(u) d \dot{u} \geq C\left(\frac{t}{x}\right)^{p_{1}} \int_{0}^{x} \omega(u) d u, \quad \text { for } \quad x \geq t .
$$

If $C_{p_{1}}(\omega)$ is the maximal $C$ for which (3.6) holds, then

$C_{p_{1}}(\omega) \geq \frac{1}{2 B_{p}(\omega)+1}$ for $p_{1}>\frac{2 B+1}{2 B+2} p$ and $B_{p}(\omega) \leq \frac{p}{C_{p_{1}}(\omega)\left(p-p_{1}\right)}$.

Proof: Suppose first that $\omega \in B_{p}$ and put $B_{p}(\omega)=B$. By the preceding lemma we know that, for $p_{1}=\frac{2 B+1}{2 B+2} p<p, \omega \in B_{p_{1}}$ with constant $2 B$. Thus

$$
\begin{aligned}
2 B \int_{0}^{x} \omega(u) d u \geq x^{p_{1}} \int_{x}^{\infty} \frac{\omega(u)}{u^{p_{1}}} d u & \geq \sum_{k=0}^{\infty} 2^{-(k+1) p_{1}} \int_{x 2^{k}}^{x 2^{k+1}} \omega(u) d u \geq \\
& \geq \sum_{k=0}^{N-1} 2^{-(k+1) p_{1}} \int_{x 2^{k+1}}^{x} \omega(u) d u .
\end{aligned}
$$

This gives, for every $x>0$,

$$
\begin{aligned}
\left(1-2^{-p_{1}}\right) \sum_{k=1}^{N-1} 2^{-k p_{1}} \int_{0}^{x 2^{k}} \omega(u) d u+2^{-N p_{1}} & \int_{0}^{x 2^{N}} \omega(u) d u \leq \\
& \leq\left(2 B+2^{-p_{1}}\right) \int_{0}^{x} \omega(u) d u .
\end{aligned}
$$

Therefore, taking only the last term on the left into account and replacing $x$ by $x 2^{-N}$, we find

$$
\int_{0}^{x 2^{-N}} \omega(u) d u \geq \frac{1}{2^{N p_{1}}(2 B+1)} \int_{0}^{x} \omega(u) d u .
$$


For $x 2^{-(N+1)} \leq t \leq x 2^{-N}$ we have

$$
\begin{aligned}
\int_{0}^{t} \omega(u) d u \geq \int_{0}^{x 2^{-(N+1)}} \omega(u) d u \geq \frac{1}{2^{N p_{1}}(2 B+1)} & \int_{0}^{x} \omega(u) d u \geq \\
& \geq \frac{1}{2 B+1}\left(\frac{t}{x}\right)^{p_{1}} \int_{0}^{x} \omega(u) d u .
\end{aligned}
$$

Thereby we have proved the necessity of the condition and the first inequality between the constants.

To prove the suffiency we assume $p_{1}<p$ and

$$
\int_{0}^{t} \omega(u) d u \geq C\left(\frac{t}{x}\right)^{p_{1}} \int_{0}^{x} \omega(u) d u, \quad \text { for } \quad 0 \leq t \leq x .
$$

Multiply this inequality by $t^{-p_{1}} x^{p_{1}-1-p}$. We get

$$
\frac{1}{C t^{p_{1}} \cdot x^{1+p-p_{1}}} \int_{0}^{t} \omega(u) d u \geq \frac{1}{x^{p+1}} \int_{0}^{x} \omega(u) d u .
$$

This inequality is valid for $0 \leq t \leq x$. We integrate with respect to $x$ over the interval $(t, \infty)$ and change the order of integration in the right member. The result is

$$
\frac{1}{C \cdot\left(p-p_{1}\right) \cdot t^{p}} \int_{0}^{t} \omega(u) d u \geq \frac{1}{p} \int_{0}^{t} \frac{\omega(u)}{t^{p}} d u+\frac{1}{p} \int_{t}^{\infty} \frac{\omega(u)}{u^{p}} d u .
$$

Hence

$$
\int_{t}^{\infty} \frac{\omega(u)}{u^{p}} d u \leq \frac{p}{C \cdot\left(p-p_{1}\right)} \cdot \frac{1}{t^{p}} \int_{0}^{t} \omega(u) d u
$$

This completes the proof of the necessity and the second inequality between the constants.

We complete the analogy by

Theorem 5.

$$
B_{\infty}=\bigcup_{p>0} B_{p}
$$


Proof: Suppose $\omega \in B_{p}$ for some $p>0$. It is immediate from Theorem 4 that $\omega$ satisfies the requirements for being in $B_{\infty}$. Thus

$$
B_{\infty} \supset \bigcup_{p>0} B_{p}
$$

Suppose on the other hand that $\omega \in B_{\infty}$ with $d(\omega)=C$, i.e.

$$
\int_{0}^{2 x} \omega(t) d t \leq C \int_{0}^{x} \omega(t) d t .
$$

This means that

$$
\int_{x}^{2 x} \omega(t) d t \leq(C-1) \int_{0}^{x} \omega(t) d t .
$$

Thus

$$
\begin{gathered}
\int_{x}^{\infty} \frac{\omega(t)}{t^{p}} d t=\sum_{k=0}^{\infty} \int_{2^{k} x}^{2^{k+1} x} \frac{\omega(t)}{t^{p}} d t \leq \sum_{k=0}^{\infty} \frac{1}{2^{k p} x^{p}} \int_{2^{k} x}^{2^{k+1} x} \omega(t) d t \leq(C-1) \\
\sum_{k=0}^{\infty} \frac{1}{2^{k p} x^{p}} \int_{0}^{2^{k} x} \omega(t) d t \leq(C-1) \sum_{k=0}^{\infty} \frac{C^{k}}{2^{k p} x^{p}} \int_{0}^{x} \omega(t) d t=\frac{2^{p}(C-1)}{\left(2^{p}-C\right) x^{p}} \int_{0}^{x} \omega(t) d t
\end{gathered}
$$

for $p>\log _{2} C$. So $\omega \in B_{p}$ for $p>\log _{2} C$ and

$$
B_{\infty} \subset \bigcup_{p>0} B_{p}
$$

and the proof is complete.

\section{3. $m_{\infty}^{\prime}$ as limit case of $m_{p}^{\prime}$.}

In this section we will for convenience use a special notation, $L_{d}(\omega)$, for the set of all non-negative, non-increasing functions in $L(\omega)$.

Theorem 6. $M_{0}$ is a bounded operator on $L_{d}(\omega)$, (i.e. $m_{\infty}^{\prime}<\infty$ ), if and only if $\omega \in B_{\infty}$ and

$$
d(\omega) \leq\left(2 m_{\infty}^{\prime}(\omega)-1\right)^{2} \leq C_{0}(d(\omega))^{9.4},
$$


where $C_{0}$ is an absolute constant.

Proof of part 1: We give first a short proof of the first part of the theorem without estimates of the constants. Suppose therefore that $M_{0}$ is a bounded operator on $L_{d}(\omega)$. Since $M_{\frac{1}{p}} f(x)$ tends monotonically to $M_{0} f(x)$ as $p$ tends to infinity, it is an immediate consequence of the monotone convergence theorem that

$$
\lim _{p \rightarrow \infty} m_{p}^{\prime}(\omega)=m_{\infty}^{\prime}(\omega)<\infty
$$

Thus $m_{p}^{\prime}(\omega)<\infty$ for $p$ large enough. By [1] this implies that $\omega \in B_{p}$ for $p$ large enough and then, by Theorem $5, \omega \in B_{\infty}$.

If on the other hand $\omega \in B_{\infty}$, then, by Theorem 5 again, $\omega \in B_{p}$ for $p$ large enough and the result in [1] implies $m_{p}^{\prime}(\omega)<\infty$. Hence $m_{\infty}(\omega)<\infty$, which means that $M_{0}$ is bounded on $L_{d}(\omega)$.

We will now present a complete proof of Theorem 6 that does not rely on the results of Arino and Muckenhoupt, but is based on another technique. It has the advantage that it gives estimates of $m_{\infty}^{\prime}(\omega)$ in terms of $d(\omega)$. To complete the proof we need the following lemma.

Lemma 4. Suppose $\sum_{-\infty}^{\infty} a_{k}$ is a positive series with sum A. Form a new series with the convoluted terms

$$
b_{k}=\sum_{m=-\infty}^{\infty} \frac{a_{m}}{2^{\epsilon|k-m|}} .
$$

Then

$$
b_{k} \geq a_{k}, \quad 2^{-\epsilon} \leq \frac{b_{k+1}}{b_{k}} \leq 2^{\epsilon} \quad \text { and } \quad \sum_{k=-\infty}^{\infty} b_{k} \leq \frac{2^{\epsilon}+1}{2^{\epsilon}-1} A .
$$

Proof:

$$
b_{k}=\cdots+a_{k-2} 2^{-2 \epsilon}+a_{k-1} 2^{-\epsilon}+a_{k}+a_{k+1} 2^{-2 \epsilon}+a_{k+2} 2^{-2 \epsilon} \cdots
$$

Now the two first properties are trivial and the third follows from a change of order of summation.

Proof of Theorem 6: Suppose first that $m_{\infty}^{\prime}(\omega)=K<\infty$. Then

$$
\int_{0}^{\infty} M_{0} f(x) \omega(x) d x \leq K \int_{0}^{\infty} f(x) \omega(x) d x, \forall f \in L_{d}(\omega) .
$$


Choose $a$ in $0<a<1$ and put

$$
f(x)= \begin{cases}1, & 0<x \leq r \\ a, & r<x \leq 2 r \\ 0, & x>2 r\end{cases}
$$

Then $f \in L_{d}(\omega)$ and

$$
M_{0} f(x)= \begin{cases}1, & 0<x \leq r \\ a^{1-\frac{r}{x}}, & r<x \leq 2 r \\ 0, & x>2 r\end{cases}
$$

We apply formula (3.7) and obtain

$$
\int_{0}^{r} \omega(x) d x+\int_{r}^{2 r} a^{1-\frac{r}{x}} \omega(x) d x \leq K\left(\int_{0}^{r} \omega(x) d x+\int_{r}^{2 r} a \omega(x) d x\right) .
$$

Thus

$$
a \int_{r}^{2 r}\left(a^{-\frac{r}{x}}-K\right) \omega(x) d x \leq(K-1) \int_{0}^{r} \omega(x) d x .
$$

We choose $a=(2 K)^{-2}$. Since $\frac{r}{x} \geq \frac{1}{2}$ we obtain

$$
\int_{r}^{2 r} \omega(x) d x \leq 4 K(K-1) \int_{0}^{r} \omega(x) d x
$$

which means that $\omega \in B_{\infty}$ with doubling constant at most $(2 K-1)^{2}$. It also follows from this inequality that $K$ has to be strictly greater than 1 , otherwise $\omega$ has to be identically zero

Suppose on the other hand that $\omega \in B_{\infty}$ with doubling constant $C$. Choose the sequence $\left\{\alpha_{k}\right\}_{-\infty}^{\infty}$ such that

$$
\int_{0}^{\alpha_{k}} \omega(x) d x=C^{-k}
$$

Using the doubling property we see

$$
C^{-k}=\int_{0}^{\alpha_{k}} \omega(x) d x=C \int_{0}^{\alpha_{k+1}} \omega(x) d x \geq \int_{0}^{2 \alpha_{k+1}} \omega(x) d x .
$$


Therefore

$$
\alpha_{k} \geq 2 \alpha_{k+1}
$$

Take an arbitrary $f \in L_{d}(\omega)$ and put

$$
\int_{0}^{\infty} f(x) \omega(x) d x=K
$$

Since $f$ is non-increasing this means that

$$
K \geq \sum_{k=-\infty}^{\infty} f\left(\alpha_{k}\right) \int_{\alpha_{k+1}}^{\alpha_{k}} \omega(x) d x=\frac{C-1}{C} \sum_{k=-\infty}^{\infty} f\left(\alpha_{k}\right) C^{-k} .
$$

Now we can use Lemma 4 with $a_{k}=f\left(\alpha_{k}\right) C^{-k}$ and obtain $b_{k} \geq a_{k}$ with

$$
\sum_{-\infty}^{\infty} b_{k} \leq \frac{K C}{C-1} \frac{2^{\epsilon}+1}{2^{\epsilon}-1}
$$

We can define a new non-increasing function $g$ with $g(x) \geq f(x)$ and $g\left(\alpha_{k}\right)=C^{k} b_{k}$. Obviously $M_{0} g \geq M_{0} f$. Jensen's inequality gives

$$
\begin{aligned}
& M_{0} g\left(\alpha_{k}\right)=\exp \int_{0}^{\alpha_{k}} \ln g(x) d x \leq\left(\int_{0}^{\alpha_{k}} g^{\frac{1}{p}}(x) d x\right)^{p} \leq \\
& \leq\left(\frac{1}{\alpha_{k}} \sum_{m=k}^{\infty} g^{\frac{1}{p}}\left(\alpha_{m+1}\right)\left(\alpha_{m}-\alpha_{m+1}\right)\right)^{p} \leq\left(\frac{1}{\alpha_{k}} \sum_{m=k}^{\infty} C^{\frac{m+1}{p}} b_{m+1}^{\frac{1}{p}} \alpha_{m}\right)^{p} .
\end{aligned}
$$

By (3.8), the terms in the last series of this estimate decrease geometrically with a quotient that is at most $C^{\frac{1}{p}} 2^{\frac{\varepsilon}{p}} 2^{-1}$. Thus

$$
M_{0} f\left(\alpha_{k}\right) \leq C^{k+1} b_{k+1}\left(\frac{1}{1-C^{\frac{1}{p}} 2^{\frac{\epsilon}{p}} 2^{-1}}\right)^{p},
$$

if $p$ is large enough. We are still free to choose $\epsilon$ and $p$. We can for example choose $\epsilon=\frac{1}{2}$ and $p=3 \ln C$ if $C \geq e^{8}$. If $C<e^{8}$ we take $p=10$. Some elementary calculations then show that

$$
M_{0} f\left(\alpha_{k}\right) \leq D C^{k+1} b_{k+1} C^{3.7}
$$


where $D$ is an absolute constant. Therefore

$$
\begin{array}{r}
\int_{0}^{\infty} M_{0} f(x) \omega(x) d x \leq D \frac{C-1}{C} \sum_{k=-\infty}^{\infty} M_{0} f\left(\alpha_{k+1}\right) C^{-k} C^{k+1} b_{k+1} C^{3.7}= \\
=D C^{3.7}(C-1) \sum_{-\infty}^{\infty} b_{k} \leq E C^{4.7} K,
\end{array}
$$

where $E$ is an absolute constant. We deduce

$$
m_{\infty}^{\prime}(\omega) \leq E C^{4.7}
$$

and the theorem is proved.

Now that we have the tools, it is tempting to prove theorem (1.7) in [1], for $0<p<\infty$. We will use Theorem 4 and the technique of Theorem 6.

Theorem 7. For $0<p \leq \infty, M_{\frac{1}{p}}$ is a bounded operator on $L_{d}(\omega)$ if and only if $\omega \in B_{p}$.

Proof: $p=\infty$ is already treated in Theorem 6 .

In the easy necessity part, we have nothing new to offer. It follows directly by chosing $f=\chi_{(0, x)}$ in (3.1).

For the sufficiency part we suppose that $\omega \in B_{p}$ with $B_{p}(\omega)=B$. In Theorem 4 we take $\epsilon=\frac{p}{4(B+1)}$ and put $p_{1}=p-2 \epsilon$ and $p_{2}=p-\epsilon$. The conclusion is that $B_{p_{1}}(\omega) \leq 2 B$ and

$$
\int_{0}^{r x} \omega(u) d u \geq \frac{r^{p_{1}}}{2 B+1} \int_{0}^{x} \omega(u) d u=r^{p_{2}} \frac{r^{p_{1}-p_{2}}}{2 B+1} \int_{0}^{x} \omega(u) d u \text { for } r \leq 1 .
$$

We now choose $r_{0}<1$ so small that $r_{0}^{p_{2}-p_{1}}(2 B+1)=1$. This gives

$$
\int_{0}^{r_{0} x} \omega(u) d u \geq r_{0}^{p_{2}} \int_{0}^{x} \omega(u) d u, \quad \forall x>0 .
$$

Put $r_{0}^{p_{2}}=C_{0}^{-1}$ and choose $\left\{\alpha_{k}\right\}_{-\infty}^{\infty}$ so that $\int_{0}^{\alpha_{k}} \omega(u) d u=C_{0}^{-k}$. Then we have

$$
\int_{0}^{\alpha_{k}} \omega(x) d x=C_{0} \int_{0}^{\alpha_{k+1}} \omega(x) d x \geq \int_{0}^{\frac{\alpha_{k+1}}{r_{o}}} \omega(x) d x
$$


and therefore

$$
\alpha_{k+1} \leq r_{0} \alpha_{k} .
$$

Now we can proceed as in the proof of Theorem 6 (with $C$ replaced by $\left.C_{0}\right)$ to find

$$
M_{\frac{1}{p}} f\left(\alpha_{k}\right) \leq M_{\frac{1}{p}} g\left(\alpha_{k}\right) \leq\left(\frac{1}{\alpha_{k}} \sum_{m=k}^{\infty} C_{0}^{\frac{m+1}{p}} b_{m+1}^{\frac{1}{p}} \alpha_{m}\right)^{p} .
$$

By the definition of $C_{0}$ and (3.9) we deduce that the terms of this series decrease geometrically with a quotient that is at most $r_{0}^{1-\frac{p_{2}}{p}} 2^{\frac{\epsilon}{p}}$. We have not yet decided what $\epsilon>0$ (in Lemma 4) should be. We just have to take $\epsilon<\left(p_{2}-p\right) \frac{\ln r_{0}}{\ln 2}$ to be sure of obtaining geometrical decreasing. Take for instance $\epsilon$ equals half that quantity. Then we have

$$
M_{\frac{1}{p}} f\left(\alpha_{k}\right) \leq C(B, p) C_{0}^{k+1} b_{k+1},
$$

where $C(B, p)$ is a constant, depending only on the indicated quantities. This gives

$$
\begin{array}{r}
\int_{0}^{\infty} M_{\frac{1}{p}} f(x) \omega(x) d x \leq C(B, p) \sum_{-\infty}^{\infty} C_{0}^{k+2} b_{k+2} C_{0 i}^{-k} \leq C_{1}(B, p) K= \\
=C_{1}(B, p) \int_{0}^{\infty} f(x) \omega(x) d x
\end{array}
$$

by which we have proved the sufficiency part of the theorem.

\section{4. $A_{\infty}^{\prime}$ and non-decreasing weights.}

We end this paper by proving two theorems, the first of which is an extension to $q=\infty$ of Theorem (1.10) in [1]. The second is an analogy with Theorem 5 for non-decreasing weights $\omega$.

Theorem 8. If $\omega \in A_{\infty}^{\prime}$, then $m_{p}^{\prime}(\omega)<\infty$ for $p$ large enough.

$A$ non-decreasing $\omega$ lies in $A_{\infty}^{\prime}$ if and only if $m_{\infty}^{\prime}(\omega)<\infty$ and then $m_{\infty}^{\prime}(\omega) \geq A_{\infty}^{\prime}(\omega)$.

Theorem 9. For $\omega$ non-decreasing we have

$$
\omega \in A_{\infty}^{\prime} \Leftrightarrow \omega \in \bigcup_{p>1} A_{p}^{\prime} .
$$

The proofs of these two theorems are based on the following lemma: 
Lemma 5. Suppose that $\omega \in A_{\infty}^{\prime}$ with constant $K$. Then, for every $r>1$ there is a constant $C$, depending on $K$ and $r$, such that

$$
\int_{0}^{r x} \omega(t) d t \leq C \int_{0}^{x} \omega(t) d t
$$

For $r=2, C=4 K^{3}$ will do.

Proof: Choose an arbitrary $r>1$. For every $x>0$, the assumption and Jensen's inequality give

$$
\int_{0}^{r x} \omega(t) d t \exp \int_{0}^{r x} \ln \frac{1}{\omega(t)} d t \leq K \leq K \int_{0}^{x} \omega(t) d t \exp \int_{0}^{x} \ln \frac{1}{\omega(t)} d t .
$$

Put

$$
\int_{0}^{r x} \omega(t) d t=c \alpha \quad \text { and } \quad \int_{0}^{x} \omega(t) d t=\alpha
$$

Then

$$
\int_{x}^{r x} \omega(t) d t=(c-1) \alpha \text { and } \int_{x}^{r x} \omega(t) d t=\frac{(c-1) \alpha}{(r-1) x} .
$$

What we want to estimate is the exponential of

$$
\begin{array}{r}
\int_{0}^{r x} \ln \frac{1}{\omega(t)} d t-\int_{0}^{x} \ln \frac{1}{\omega(t)} d t=\frac{1}{r x} \int_{x}^{r x} \ln \frac{1}{\omega(t)} d t-\frac{r-1}{r x} \int_{0}^{x} \ln \frac{1}{\omega(t)} d t= \\
=\frac{r-1}{r}\left(f \ln \frac{1}{\omega(t)} d t-\int_{0}^{r x} \ln \frac{1}{\omega(t)} d t\right) .
\end{array}
$$

We now treat the two members on the left, the first by Jensen's inequality $\exp \int_{x}^{r x} \ln \frac{1}{\omega(t)} d t \geq\left(\int_{x}^{r x} \omega(t) d t\right)^{-1}=\frac{(r-1) x}{(c-1) \alpha}$ i.e. $\int_{x}^{r x} \ln \frac{1}{\omega(t)} d t \geq \ln \frac{(r-1) x}{(c-1) \alpha}$.

The second satisfies by assumption

$$
\exp \int_{0}^{x} \ln \frac{1}{\omega(t)} d t \leq \frac{K}{\alpha} x \text { i.e. } \int_{0}^{x} \ln \frac{1}{\omega(t)} d t \leq \ln K+\ln \frac{x}{\alpha} .
$$


We therefore obtain

$$
\frac{r-1}{r}\left(\int_{x}^{r x} \ln \frac{1}{\omega(t)} d t-\int_{0}^{x} \ln \frac{1}{\omega(t)} d t\right) \geq \frac{r-1}{r} \ln \frac{r-1}{K(c-1)}
$$

and inequality (3.10) gives

$$
\operatorname{cr}\left(\frac{r-1}{K(c-1)}\right)^{\frac{r-1}{r}} \leq K,
$$

or

$$
\operatorname{cr}\left(\frac{r-1}{c-1}\right)^{\frac{r-1}{r}} \leq K^{2-\frac{1}{r}} .
$$

For any $r>1$ we see that $c$ cannot be arbitrarily large, but has to be smaller than some number, which depends on $r$ and $K . \quad r=2$, for example, gives the doubling constant $d(\omega)<\frac{K^{3}}{4}$. This proves the lemma.

Proof of Theorem 8: Suppose that $\omega \in A_{\infty}^{\prime}$ with constant $K$. By Lemma $5, \omega \in B_{\infty}$ with $d(\omega) \leq \frac{K^{3}}{4}$. By Theorem $6, m_{\infty}^{\prime}(\omega)<\infty$.

Suppose now that $\omega$ is a non-decreasing function with finite $m_{\infty}^{\prime}(\omega)$. Then we use the inequality

$$
\int_{0}^{\infty} M_{0} f(t) \omega(t) d t \leq m_{\infty}^{\prime}(\omega) \int_{0}^{\infty} f(t) \omega(t) d t
$$

with the non-increasing function $f=\frac{1}{\omega} \chi(0, x)$ to obtain

$$
\int_{0}^{x} \exp \left(\int_{0}^{t} \ln \frac{1}{\omega(s)} d s\right) \omega(t) d t \leq m_{\infty}^{\prime}(\omega) x
$$

Since $\omega$ is non-decreasing and $t \leq x$ in the integration

$$
\int_{0}^{t} \ln \frac{1}{\omega(s)} d s \geq \int_{0}^{x} \ln \frac{1}{\omega(s)} d s
$$

This gives

$$
\int_{0}^{x} \omega(t) d t \exp \int_{0}^{x} \ln \frac{1}{\omega(t)} d t \leq m_{\infty}^{\prime}(\omega)
$$


and thus

$$
A_{\infty}^{\prime}(\omega) \leq m_{\infty}^{\prime}(\omega) .
$$

Thereby we have proved Theorem 8 .

Proof of Theorem 9: This is now more or less a corollary. By Jensen's inequality $A_{\infty}^{\prime}(\omega) \leq A_{p}^{\prime}(\omega)$ and therefore $A_{p}^{\prime} \subset A_{\infty}^{\prime}, \forall p>1$. On the other hand, by Lemma 5 and Ariño-Muckenhoupt's result

$$
\omega \in A_{\infty}^{\prime} \Rightarrow\left\{\omega \in B_{p} \quad \text { for some } p>1\right\} \Rightarrow \omega \in A_{p}^{\prime} .
$$

Therefore, for non-decreasing $\omega, A_{\infty}^{\prime} \subset \bigcup A_{p}^{\prime}$ and the proof is complete.

It is natural to ask whether $\omega \in A_{\infty}^{\prime}$ implies $\omega \in A_{p}^{\prime}$ for some $p>1$, i.e. if Theorem 8 could be strengthened to comprise also the case of weight functions that are not non-decreasing. This, however, is not true. We can for example take

$$
\omega(x)= \begin{cases}\exp -\frac{1}{\sqrt{(1-x)}}, & 0<x<1, \\ 1, & x \geq 1 .\end{cases}
$$

This function clearly lies in $A_{\infty}^{\prime}$ but not in $A_{p}^{\prime}$ for any $p>1$, but it is easy to see that $B_{p}(\omega)$ is finite for every $p>1$ and therefore $m_{p}^{\prime}(\omega)<\infty$ for every $p>1$. This example also shows that $A_{\infty}^{\prime}(\omega) \neq \lim _{p \rightarrow \infty} A_{p}^{\prime}(\omega)$.

\section{References}

1. A. Ariño And B. Muckenhoupt, Maximal functions on classical Lorentz spaces and Hardy's inequality with weights for non increasing functions, Trans. Amer. Math. Soc. 320(2) (1990), 727-735.

2. S. M. BuCKLeY, Harmonic Analysis on weighted spaces, PhD-thesis, Chicago, 1990.

3. I. Garcia-Cuerva and I. L. Rubio de Francia, "Weighted norm inequalities and related topics," North Holland, 1985.

4. G. H. Hardy, J. E. Littlewood and G. Polya, "Inequalities," Cambridge Univ. Press, 1951.

5. S. V. HRUŠČEV, A description of weights satisfying the $A_{\infty}$-condition of Muckenhoupt, Proc. Amer. Math. Soc. 90 (1984), 253-257.

6. R. L. Johnson, Changes of variable and $A_{p}$-weights, Contemporary Math. 107 (1990), 93-99. 
7. B. MuCKenhoupt, Weighted norm inequalities for the Hardy maximal function, Trans. Amer. Math. Soc. 165 (1972), 207-228.

8. J. O. Strömberg AND A. TORChinsKy, "Weighted Hardy Spaces," Lecture notes in math. 1381, Springer Verlag, Berlin, 1989.

9. A. Torchinsky, "Real variable methods in Harmonic Analysis," Acad. Press, London, 1988.

10. I. WIK, On Muckenhoupt's classes of weight functions, Studia Math. 94 (1989), 245-255.

Carlo Sbordone:

Dipartimento di Matematica

e Applicazioni "R. Caccioppoli"

Università Via Cintia

80126 Napoli

ITALY
Ingemar Wik:

Department of Mathematics

University of Umeå

90187 Umeå

SWEDEN

Rebut el 25 d'Agost de 1993 